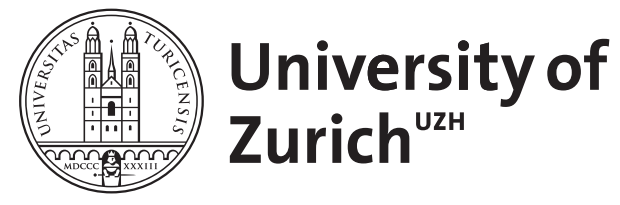
Archive

University of Zurich

University Library

Strickhofstrasse 39

CH-8057 Zurich

www.zora.uzh.ch

Year: 2021

Wieder mehr Wirtschaftsgeschichte in der Schweiz im 19. Jahrhundert - ein Plädoyer

Straumann, Tobias

DOI: https://doi.org/10.24894/2296-6013.00079

Posted at the Zurich Open Repository and Archive, University of Zurich ZORA URL: https://doi.org/10.5167/uzh-203331

Journal Article

Published Version

Originally published at:

Straumann, Tobias (2021). Wieder mehr Wirtschaftsgeschichte in der Schweiz im 19. Jahrhundert - ein Plädoyer. Schweizerische Zeitschrift für Geschichte (Revue suisse d'histoire), 71(1):143-158.

DOI: https://doi.org/10.24894/2296-6013.00079 


\section{Wieder mehr Wirtschaftsgeschichte der Schweiz im 19. Jahrhundert - ein Plädoyer}

Tobias Straumann

Bis in die 1990er Jahre war das 19. Jahrhundert eine der beliebtesten Zeitperioden der schweizerischen Wirtschaftsgeschichte. ${ }^{1}$ Vor allem der Prozess der Industrialisierung, der Wirtschaft und Gesellschaft von Grund auf veränderte, wurde in all seinen Schattierungen intensiv erforscht und ausgemessen. Dann aber flachte das Interesse spürbar ab, und das 20. Jahrhundert rückte in den Vordergrund. Ausdruck des neuen Forschungsmittelpunktes war die Publikation des Handbuches «Wirtschaftsgeschichte der Schweiz im 20. Jahrhundert» im Jahr 2012. ${ }^{2}$ Natürlich kam das Interesse am 19. Jahrhundert nie ganz zum Erliegen, dafür ist die Zeitperiode zu wichtig. Aber die einst populären Themen wurden zunehmend an den Rand gedrängt, und an den internationalen Debatten war die Schweizer Historiographie kaum noch beteiligt, während sie früher an vorderster Stelle vertreten war.

Es scheint mir deshalb dringlich, bald das Projekt einer neuen Schweizer Wirtschaftsgeschichte des 19. Jahrhunderts zu lancieren. Es ginge dabei nicht nur darum, das vorhandene Wissen wieder in Erinnerung zu rufen, sondern auch das reichhaltige statistische Material mit neuen Methoden und Werkzeugen zu nutzen, neue Forschungsarbeiten anzuregen und dadurch den Anschluss an die internationalen Forschungstrends zu verbessern. Im Folgenden möchte ich dazu einige Anregungen geben, indem ich zehn Forschungsfragen identifiziere, die meines Erachtens für eine internationale Einbettung der Wirtschaftsgeschichte der Schweiz im 19. Jahrhundert zentral sind. Selbstverständlich ist die Liste der Themen und der erwähnten Literatur alles andere als vollständig. Sie soll lediglich als Basis für eine weiterführende Diskussion dienen.

\section{Die Ursachen der Industrialisierung}

Die Frage, warum gewisse Länder im 19. Jahrhundert wirtschaftlich erfolgreich waren beziehungsweise wurden, während andere arm blieben, ist wohl die wichtigste Frage der Wirtschaftsgeschichte. Die Schweiz ist in diesem Kontext interessant, weil sie früh mit Erfolg auf den technologischen Druck, der von der industriellen Revolution in England ausging, reagieren konnte. Zwischen 1806 und

1 Ich danke der Alfred Escher-Stiftung für die Diskussionen und den finanziellen Beitrag für die Erarbeitung des vorliegenden Textes und Dr. Beat Gnädinger, Stiftungsratsmitlied und Staatsarchivar des Kantons Zürich, für die ideelle Unterstützung.

2 Patrick Halbeisen, Margrit Müller und Béatrice Veyrassat (Hg.), Wirtschaftsgeschichte der Schweiz im 20. Jahrhundert, Basel 2012. 
1813 erhöhte sich die Zahl der mechanischen Baumwollspinnereien von 6 auf knapp 100 - davon befanden sich 66 im Kanton Zürich, 17 im Kanton St. Gallen und 7 im Kanton Appenzell Ausserrhoden, das heisst in jenen Gebieten, wo schon im 18. Jahrhundert die protoindustrielle Baumwollverarbeitung weit verbreitet gewesen war. Bereits Ende der 1820er Jahren importierte die Schweiz kaum noch Baumwollgarn, und in den 1840er Jahren wurden die Schweizer Firmen bei fast allen Garnsorten konkurrenzfähig.

Dank einer Reihe von älteren grundlegenden Arbeiten sind viele Fragen weitgehend beantwortet. ${ }^{3}$ So kennen wir die soziale Herkunft der Unternehmer, die Quellen des Investitionskapitals, das meist nicht aus den Kreisen des Handelskapitals stammte, oder die Art und Weise, wie die ersten Firmen Technologien vom Ausland übernommen und weiter entwickelt haben. Wir wissen auch viel über die Arbeitsbedingungen der Arbeiterinnen und Arbeiter und ihrer Kinder, über Fabrikordnungen oder den Pauperismus der 1830er und 1840er Jahre. Es gibt noch vieles zu erforschen, aber der Kenntnisstand ist in diesen Themenfeldern hoch.

Ungeklärt ist aber die Frage geblieben, inwiefern die durch die französische Besetzung erfolgte Einführung der Handels- und Gewerbefreiheit und die napoleonische Kontinentalsperre die frühe Industrialisierung der Schweiz ermöglicht haben. Die zeitliche Dimension ist dabei unbestritten. Wie erwähnt, nahm die Zahl der Spinnereien genau in der Zeit der Kontinentalsperre von 1806 bis 1813 sprunghaft $\mathrm{zu}$, und viele der Firmengründungen wären zweifellos vor dem französischen Einmarsch von 1798 nicht möglich gewesen, weil rechtliche Schranken die Landbevölkerung benachteiligten. Die Frage ist vielmehr, ob die Industrialisierung der Schweiz ohne Kontinentalsperre so früh hätte stattfinden können. Der deutsche Entwicklungssoziologe Ulrich Menzel ist der Meinung, dass ohne die vorübergehende Abschirmung von der englischen Konkurrenz und ohne die Einführung der Handels- und Gewerbefreiheit die Gefahr bestanden hätte, dass die Schweiz sich in einen armen Agrarstaat verwandelte. ${ }^{4}$ Ulrich Pfister hingegen

3 Wichtige Grundlagenwerke zum Beginn der Industrialisierung in der Schweiz sind Walter Bodmer, Die Entwicklung der schweizerischen Textilwirtschaft im Rahmen der übrigen Industrien und Wirtschaftszweige, Zürich 1960; Rudolf Braun, Sozialer und kultureller Wandel in einem ländlichen Industriegebiet (Zürcher Oberland) unter Einwirkung des Maschinen- und Fabrikwesens im 19. und 20. Jahrhundert, Erlenbach-Zürich 1965; Béatrice Veyrassat, Négociants et fabricants dans l'industrie cotonnière suisse 1760-1840, Lausanne 1982; Albert Tanner, Spulen - Weben - Sticken. Die Industrialisierung in Appenzell Ausserrhoden, Zürich 1982; Jean-François Bergier, Wirtschaftsgeschichte der Schweiz, Zürich 1983; Reto Jäger, Max Lemmenmeier, August Rohr und Peter Wiher, Baumwollgarn als Schicksalsfaden. Wirtschaftliche und gesellschaftliche Entwicklungen in einem ländlichen Industriegebiet (Zürcher Oberland) 1750-1920, Zürich 1986; Peter Dudzik, Innovation und Investition. Technische Entwicklung in der schweizerischen Baumwollspinnerei 1800 bis 1916, Zürich 1987.

$4 \quad$ Ulrich Menzel, Auswege aus der Abhängigkeit. Die entwicklungspolitische Aktualität Europas, Frankfurt 1988, S. 45. 
vermutet, dass die lange protoindustrielle Tradition stärker zu gewichten sei als die kurze Phase des Protektionismus. ${ }^{5}$

Um die Gewichtung der verschiedenen Faktoren zu verfeinern, wäre es wichtig, die schweizerische Erfahrung international einzubetten und zu vergleichen, denn nicht nur in der Schweiz, sondern auch im französischen besetzten Rheinbund oder in den Vereinigten Staaten fand hinter schützenden Zollmauern eine frühe Entwicklung der mechanischen Baumwollspinnerei statt. Kevin O’Rourke hat versucht, die internationalen Barrieren während der napoleonischen Kriege zu messen, indem er die Preisentwicklung von inländisch produzierten Rohstoffen und Gütern mit derjenigen der importierten Rohstoffe und Güter verglich. ${ }^{6}$ Daten für die Schweiz, die international vergleichbar sind, fehlen allerdings. Es wäre wichtig, die Kontinentalsperre quantitativ zu messen, um bei einer der wesentlichen Fragen der Industrialisierungsforschung mitreden zu können. Es geht ganz grundsätzlich um die alte Frage des deutschen Ökonomen Friedrich List: Wie stark muss sich ein Land vor der internationalen Konkurrenz schützen dürfen, um eine neue Industrie aufzubauen?

\section{Das Industrialisierungsmuster der Schweiz}

Obwohl die Industrialisierung der Schweiz früh einsetzte, blieben die Löhne fast das ganze 19. Jahrhundert hindurch relativ niedrig. Zuletzt hat Roman Studer diesen Sachverhalt nachgewiesen, indem er die Löhne von ungelernten Bauarbeitern in europäischen Städten miteinander verglich. ${ }^{7}$ Studers Daten zeigen, dass eine Verbesserung der schweizerischen Reallöhne dieser Berufsgruppe erst in den 1880er Jahren einsetzte. Von da an war das Lohnwachstum hingegen überdurchschnittlich, so dass die Schweiz am Vorabend des Ersten Weltkriegs zu den reicheren Ländern des Kontinents gehörte. Diese Entwicklung zeigen auch die neusten Daten von Christian Stohr. ${ }^{8}$

$5 \quad$ Ulrich Pfister, Protoindustrialisierung, in: Historisches Lexikon der Schweiz online, Version 20.8. 2013: https://hls-dhs-dss.ch/de/articles/013823/2013-08-20/ (1.12.2019). Vgl. Pfisters Grundlagenwerk: Ulrich Pfister, Die Zürcher Fabriques. Protoindustrielles Wachstum vom 16. zum 18. Jahrhundert, Zürich 1992.

6 Kevin O'Rourke, The worldwide economic impact of the French Revolutionary and Napoleonic Wars, 1793-1815, in: Journal of Global History 1 (2006), S. 123-149.

7 Roman Studer, When did the Swiss get so rich? Comparing living standards in Switzerland and Europe, 1800-1913, in: Journal of European economic history (2008), S. 405-451.

8 Christian Stohr, Trading gains. New estimates of Swiss GDP, 1851 to 2008, in: Working Papers of the Paul Bairoch Institute of Economic History 4 (2016). Vgl. ausgewählte frühere Arbeiten zum Lebensstandard im späten 19. Jahrhundert: Erich Gruner und Hans-Rudolf Wiedmer, Demographische, wirtschaftliche und soziale Basis und Arbeitsbedingungen, Arbeiterschaft und Wirtschaft in der Schweiz 1880-1914, Band 1, Zürich 1987; Jakob Tanner, Fabrikmahlzeit. Ernährungswissenschaft, Industriearbeit und Volksernährung in der Schweiz, 1890-1950, Zürich 1999. 
Allerdings wissen wir immer noch wenig über die tatsächlichen Lebensverhältnisse der Menschen, ihre Löhne, ihre zusätzlichen Einkünfte und ihre Konsummuster in der ersten Hälfte des 19. Jahrhunderts. Je nach Branche, Region, Alter, Geschlecht und familiären Verhältnissen variierte das effektive Einkommen stark. Möglicherweise muss sogar die heute vorherrschende Meinung, dass die Schweizer Löhne im internationalen Vergleich tief waren, relativiert werden. Das würde auch die Diskussion über das Industrialisierungsmuster neu lancieren.

Bisher ist man nämlich davon ausgegangen, dass tiefe Löhne in der Textilindustrie, dem deutschschweizerischen Leitsektor des 19. Jahrhunderts, notwendig waren, um im Export erfolgreich zu sein. ${ }^{9}$ Wohl bemühten sich die Spinnereien und Webereien, möglichst hochwertige Garnsorten und Tücher zu produzieren, bei denen eine hohe Marge erzielt werden konnte. Aber die hohen Transportkosten, die Zölle in Europa und der intensive internationale Wettbewerb erlaubten es nicht, die Löhne über ein bescheidenes Niveau hinaus anzuheben. In dieser Hinsicht gleicht das schweizerische Industrialisierungsmuster demjenigen der ostasiatischen Länder (Japan, Südkorea und Taiwan), die auch nur dank tiefer Löhne eine Textilindustrie aufbauen konnten. Ganz anders verlief die Entwicklung in den USA. Die Knappheit an Arbeitskräften im Verhältnis zum grossen Land und der im Zuge der hohen Immigrationsraten im 19. Jahrhundert schnell wachsende Binnenmarkt führten dazu, dass das Lohnniveau relativ hoch war und die eigene Industrie mit protektionistischen Massnahmen von der ausländischen Konkurrenz abgeschirmt und entwickelt werden konnte.

Ein vertieftes Verständnis des schweizerischen Industrialisierungsmusters würde eine Weiterführung des regionalen Vergleichs in Europa erfordern. Das Standardwerk von Sidney Pollard ist 1981 erschienen, aber hat wenig Nachfolger gefunden. ${ }^{10}$ Eine Belebung dieses äusserst ergiebigen Zugangs zur europäischen Wirtschaftsgeschichte ist erst in jüngster Zeit wieder spürbar geworden, und die neuste Publikation konzentriert sich auf das 20. Jahrhundert."1 Interessant wäre in diesem Zusammenhang nicht nur der Vergleich der Industrieregionen, sondern auch der landwirtschaftlichen Gebiete. Diesbezüglich gibt es nur für den Alpenraum überregionale Darstellungen. ${ }^{12}$ Die Landwirtschaft des Mittellandes im 19. Jahrhundert ist hingegen kaum aus vergleichender Perspektive untersucht

9 Veyrassat, Négociants et fabricants, S. 28 ff. Vgl. O’Rourke, Williamson, Globalization and History.

10 Sidney Pollard, Peaceful Conquest. The Industrialization of Europe 1760-1970, Oxford 1981.

11 Joan R. Rosés und Nikolaus Wolf (Hg.), The Economic Development of Europe's Regions. A Quantitative History since 1900, London 2019.

12 Jon Mathieu, Die Alpen. Raum - Kultur - Geschichte, Stuttgart 2015. Vgl. die laufenden Publikationen des Laboratorio di Storia delle Alpi (LabiSAlp) und die Serie «Histoire des Alpes» seit 1996. 
worden. ${ }^{13}$ Besonders interessant ist dabei die Herauslösung der schweizerischen Bauernbetriebe aus den komplizierten Rechtsverhältnissen des Ancien Régime, die überall in Europa stattfand. Aufschlussreich wäre ferner eine vergleichende Untersuchung des Agrarprotektionismus, der in den 1870er Jahren einsetzte. Die Grundzüge sind bekannt, aber ein genaueres Verständnis würde eine detaillierte Untersuchung der Zölle für jedes einzelne Agrargut im internationalen Kontext erfordern. ${ }^{14}$

\section{Globalisierung}

Die Schweizer Wirtschaft hätte nie erfolgreich sein können, wenn sie nicht die Vorteile des Weltmarkts hätte nutzen können. Der Binnenmarkt ist zu klein, und die natürliche Ressourcenausstattung zu schwach, um eine schnell wachsende Bevölkerung ernähren zu können. Früh haben sich deshalb die verschiedenen Regionen auf ihre Stärken spezialisiert, um sich durch Export wichtige Güter verschaffen zu können. Im späten 19. Jahrhundert nahm die schweizerische Integration ein Ausmass an, das mit den heutigen Verhältnissen vergleichbar ist. Nicht nur Güter, Dienstleistungen und Kapital wurden über die Grenzen transferiert, es fanden auch grosse Wanderungsbewegungen aus der Schweiz und in die Schweiz statt. ${ }^{15}$ Neuere Arbeiten haben ferner bestätigt, dass die Schweizer Wirtschaft in vielfältiger Weise mit Kolonialreichen und Rohstoffländern, in denen verschiedene Formen unfreier Arbeit verbreitet waren, verbunden war. ${ }^{16}$

13 Eine grundlegende Studie ist Jakob Stark, Zehnten statt Steuern. Das Scheitern der Ablösung von Zehnten und Grundzinsen in der Helvetik: Eine Analyse des Vollzugs der Grundlasten- und Steuergesetze am Beispiel des Kantons Thurgau, Zürich 1993.

14 Cédric Humair, Développement économique et état central (1815-1914). Un siècle de politique douanière Suisse au service des élites, Bern 2004. Für Deutschland liegt eine Untersuchung vor, die für die Analyse der schweizerischen Situation genutzt werden könnte: Sibylle Lehmann, The German elections in the 1870s: who caused the turn towards protectionism?, in: Journal of Economic History 70 (2010), S. 146-178.

15 Für einen gesamtschweizerischen Überblick siehe Béatrice Veyrassat, Wirtschaft und Gesellschaft an der Wende zum 20. Jahrhundert, in: Patrick Halbeisen, Margrit Müller und Béatrice Veyrassat, Wirtschaftsgeschichte der Schweiz im 20. Jahrhundert, Basel 2012, S. 33-81. Für die umfangreiche Tessiner Auswanderung siehe Luigi Lorenzetti, Economie et migrations au XIX siècle: les stratégies de la reproduction familiale au Tessin, Bern 1999. Internationale Standardwerke sind Kevin O'Rourke und Jeffrey Williamson, Globalization and History. The Evolution of a Nineteenth-Century Atlantic Economy, Cambridge (Mass.) 1999; Michael Bordo, Alan Taylor und Jeffrey Williamson (Hg.), Globalization in Historical Perspective, Chicago 2003.

16 Eine neue Übersicht bieten Béatrice Veyrassat, Histoire de la Suisse et des Suisses dans la marché du monde (XVII siècle - Première guerre mondiale). Espaces - circulations - échanges, Neuchâtel 2018; Lea Haller, Transithandel. Geld- und Warenströme im globalen Kapitalismus, Frankfurt a. M. 2019. Ältere Werke sind Niklaus Stettler, Peter Haenger und Robert Labhardt, Baumwolle, Sklaven und Kredite. Die Basler Welthandelsfirma Christoph Burckhardt \& Cie. in revolutionärer Zeit (1789-1815), Basel 2004; Thomas David, Bouda Etemad und Janick Marina Schaufelbuehl, La Suisse et l'esclavage des Noirs, Lausanne 2005; Andrea Franc, Wie die Schweiz zur Schokolade kam. Der 
Die grossen Etappen der Integration sind bekannt, aber im Einzelnen wissen wir wenig über die internationalen Wertschöpfungsketten, in denen sich die Schweizer Unternehmen einreihten. ${ }^{17}$ Zur Grunderfahrung eines konstanten Wandels kamen periodische Wirtschaftskrisen und kriegerische Ereignisse hinzu. Der Amerikanische Bürgerkrieg (1861-65) verknappte zum Beispiel die globale Baumwollversorgung, und der Deutsch-Französische Krieg (1870-71) löste eine Liquiditätskrise bei den Schweizer Banken aus.

Die Globalisierung des 19. Jahrhunderts ist auch deswegen interessant für die aktuellen Debatten, weil in den europäischen Ländern bereits damals heftig darüber diskutiert wurde, wie sich eine Wirtschaft partiell vor der ausländischen Konkurrenz schützen kann und welche Kompensationen ein Staat anbieten muss, um die Unterstützung der Bevölkerung für die Globalisierung aufrechtzuerhalten. Die Schweiz ist in diesem Zusammenhang interessant, weil sie im späten 19. Jahrhundert zur Gruppe der besonders exponierten Länder gehörte. ${ }^{18} \mathrm{Bei}$ den Industriegütern und dem Getreideimport blieb die Schweiz liberal, während sie beim Fleischimport und den Milchprodukten gezielt Zölle erhöhte. Wie sich Protektionismus und Freihandel nach Region, Beruf, Geschlecht und Alter in der Schweiz auswirkte, wäre eine interessante Fragestellung.

Was die Rekrutierung der Fachkräfte anbelangt, so lässt sich gegen Ende des 19. Jahrhundert eine Stärkung des einheimischen Nachwuchses feststellen, nachdem noch bis in die 1870er Jahre viele Firmengründer und bestimmenden Manager aus dem Ausland geholt worden waren. Dies geschah einerseits über den Ausbau der Hochschulen und der übrigen höheren Bildung, anderseits über die auf Langfristigkeit angelegte Nachwuchsförderung innerhalb der Firmen. Damit gelang es, eine bessere Balance zwischen ausländischen Fachkräften, die weiterhin unverzichtbar für die Schweizer Wirtschaft blieben, und den Einheimischen herzustellen, was die Kontroversen um die Einwanderungs- und Einbürgerungspolitik ein Stück weit entspannte. Über diesen Prozess ist bisher kaum geforscht worden. ${ }^{19}$

Kakaohandel der Basler Handelsgesellschaft mit der Kolonie Goldküste (1893-1960), Basel 2008; Christof Dejung, Unbekannte Intermediäre. Schweizerische Handelsfirmen im 19. und 20. Jahrhundert, in: Schweizerische Zeitschrift für Geschichte 17 (2010), S. 139-155; Andreas Zangger, Koloniale Schweiz. Ein Stück Globalgeschichte zwischen Europa und Südostasien, Bielefeld 2011.

17 Michael Bernegger, Die Schweiz und die Weltwirtschaft: Etappen der Integration im 19. und 20. Jahrhundert; in: Paul Bairoch, Martin Körner (Hg.): Die Schweiz in der Weltwirtschaft, Schweizerische Gesellschaft für Wirtschafts- und Sozialgeschichte, Band 8, Zürich 1990, S. 429-464; Andrea Franc, Im Austausch mit der Welt: Schweizer Unternehmen im 19. und 20. Jahrhundert, Baden 2020. 18 Michael Huberman und Wayne Lewchuk, European Economic Integration and the Labour Compact, 1850-1913, in: European Review of Economic History 7 (2003), S. 3-41. Ein Pionierwerk ist William Rappard, La révolution indutrielle et les origines de la protection légale du travail en Suisse, Genf 2008.

19 Tobias Straumann, Die Schöpfung im Reagenzglas. Eine Geschichte der Basler Chemie (18501920), Basel 1995; Luca Froelicher, Corporate Governance und Krisenresistenz. Schweizerische Versicherungsunternehmen in der Zwischenkriegszeit 1919-1939, Dissertation ETH Zürich 2019. 


\section{Technologischer Fortschritt, Strukturwandel und Arbeitsmarkt}

Im 19. Jahrhundert war der Strukturwandel besonders schnell. $\mathrm{Zu}$ Beginn des Jahrhunderts arbeitete die grosse Mehrheit der Bevölkerung in der Landwirtschaft, am Vorabend des Ersten Weltkriegs waren es weniger als dreissig Prozent, und im zweiten und dritten Sektor verschwanden viele Berufe im Zuge der Industrialisierung. Wie die Entwicklung auf der aggregierten Ebene verlief, wissen wir seit dem Aufsatz Francesco Kneschaurek von 1964 recht gut. ${ }^{20}$ Erstaunlicherweise wissen wir aber wenig Konkretes, das über die aggregierten Daten hinausgeht. Wir sehen zwar, wie sich zum Beispiel die Arbeitskräfte innerhalb und zwischen den Sektoren verschoben und wie sich die Binnenwanderung und die Auswanderung entwickelten, aber wenn es darum geht $\mathrm{zu}$ verstehen, wie die Menschen damals den Strukturwandel konkret erfahren und bewältigt haben, mangelt es an Forschung. Ausnahmen bestätigen die Regel. So hat Rudolf Braun in seiner zweibändigen Studie über Industrialisierung und Volksleben im Zürcher Oberland den Wandel der Lebenswelten anschaulich beschrieben, und Karin Baumann hat in ihrer Lizenziatsarbeit den Arbeitsmarkt in der Region von Schönenwerd, wo die Firma Bally domiziliert ist, untersucht. ${ }^{21}$

Interessant wäre zum Beispiel zu wissen, wer eine Auswanderung einer Binnenwanderung in die schweizerischen Industriezentren vorgezogen hat. Man könnte die Herkunft der Arbeitskräfte auf Betriebsebene anhand von Personallisten eruieren. Wer blieb wie lange? Wer war mit wem verwandt? Was passierte mit den entlassenen Arbeitskräften? Wie war die Abhängigkeit von der Konjunktur nach Region, Herkunft, Ausbildung, Geschlecht und Alter? Da es im 19. Jahrhundert noch keine staatliche Arbeitslosenversicherung gab, ist die Erforschung dieser Fragen mit grossem Aufwand verbunden, aber angesichts der Relevanz der Fragestellung würde es sich lohnen, einschlägige Regionalstudien durchzuführen. Eine vielversprechende Quelle könnten die Akten regionaler oder kommunaler Arbeitsvermittlungen sein, die in Gemeinde- und Kantonsarchiven aufbewahrt werden.22

20 Francesco Kneschaurek, Wandlungen der schweizerischen Industriestruktur seit 1800, in: Schweizerische Zeitschrift für Volkswirtschaft und Statistik 100 (1964), S. 133-166.

21 Braun, Industrialisierung und Volksleben. Karin Baumann, Arbeitswelt, Arbeitsorganisation und regionaler Arbeitsmarkt. Die Bally Schuhfabriken 1870-1910 in Schönenwerd, Lizenziat Universität Zürich 1993.

22 Siehe dazu https://www.geschichtedersozialensicherheit.ch/institutionen/kantonale-lokale-undprivate-institutionen/arbeitsaemter (12.01.2021). 


\section{Innovationskultur}

Die Schweiz ist heute mit ihrer lebendigen KMU-Landschaft, den internationalen Grosskonzernen, den guten Hochschulen und dem dualen Bildungssystem gut aufgestellt. Aber es wäre falsch zu glauben, dass hier keinerlei Anstrengungen mehr nötig wären. Reiche Länder laufen immer Gefahr, ihren unternehmerischen Elan einzubüssen und nur mehr von den Renteneinkünften des angehäuften Vermögens zu leben. Ein frischer Blick auf das 19. Jahrhundert wäre unter diesem Aspekt erkenntnisreich.

Was die grossen unternehmerischen Leistungen des 19. Jahrhunderts anbelangt, so sind wir gut orientiert. Die Reihe «Pioniere der Wirtschaft und Technik» hat seit Mitte der 1950er Jahre mehr als hundert Bände mit Biographien wichtiger Akteure publiziert. Dazu kommen viele Firmenschriften, die anlässlich von Jubiläen erschienen sind. Und schliesslich gibt es grundlegende Studien, die die Innovationskultur von einzelnen Firmen und Branchen untersucht haben. Besonders hervorzuheben sind die Dissertationen von Peter Dudzik und Hannes Siegrist. ${ }^{23}$

In einem nächsten Schritt wäre es wichtig, die Rekrutierungsmuster und Netzwerke systematischer zu untersuchen, wie es von der Datenbank Obelis für das 20. Jahrhundert geleistet wurde. ${ }^{24}$ Woher kamen die Unternehmer und Manager? Welchen Beruf hatten ihre Eltern? Wie heirateten sie? Welche Ausbildung genossen sie? In welchem Alter gründeten sie eine Firma oder übernahmen sie eine leitende Funktion?

Des Weiteren wäre es interessant, anhand von Fallbeispielen die informellen Innovationsprozesse genauer zu untersuchen. Woher kamen die Neuerungen genau - aus dem Labor, aus der Werkstatt oder vom Kunden? Wie wichtig war die formale Bildung für innovatives Verhalten? Auf welche Weise förderten Firmeninhaber und Manager die betriebseigene Innovationskultur? Wie wichtig ist die Finanzierungsseite? Welche Projekte hatten Erfolge, welche scheiterten? Mithilfe dieser Fragen sollte es möglich sein, die wichtigsten Elemente des blühenden Innovationsbiotops des 19. Jahrhunderts zu identifizieren und in die aktuellen Diskussionen um Innovationsparks, Technologieförderung und steuerliche Anreize für Venture Capital einzubringen.

Schliesslich gilt es auch die Rolle der regulatorischen Rahmenbedingungen genauer unter die Lupe zu nehmen. Auf die Bedeutung der Handels- und Gewerbefreiheit zur Zeit der Helvetik und der Mediationsverfassung ist schon

23 Peter Dudzik, Innovation und Investition: Technische Entwicklung und Unternehmerentscheide in der schweizerischen Baumwollspinnerei 1800 bis 1896, Zürich 1987; Hannes Siegrist, Vom Familienbetrieb zum Managerunternehmen. Angestellte und industrielle Organisation am Beispiel der Georg-Fischer-AG in Schaffhausen 1797-1930, Göttingen 1981.

24 Das Projekt ist an der Universität Lausanne angesiedelt: https://www.unil.ch/obelis/en/home. html (12.01.2021). 
hingewiesen worden. Als die liberalen Kantone in den 1830er Jahren diese Freiheiten in der Verfassung verankerten, kam es wiederum zu einer Welle von Firmengründungen auf dem Land, etwa in der Seidenindustrie am linken Zürichseeufer, wodurch die Zürcher Wirtschaftsgeschichte eine neue Wendung erhielt. Ganz grundsätzlich gibt es fast keine Studien zur Geschichte der Marktverfassung und Marktzutrittsbarrieren im 19. Jahrhundert. ${ }^{25}$

\section{Institutionen und Wirtschaftswachstum}

Seit dem Erscheinen des Buches «Why nations fail» von Daron Acemoglu und James Robinson im Jahr 2012 ist die Frage nach dem Zusammenhang zwischen Institutionen und Wirtschaftswachstum ins Zentrum der internationalen Forschungsdiskussion gerückt. Die Frage ist in der schweizerischen Historiographie freilich schon viel länger diskutiert worden, vor allem dank der grundlegenden Arbeiten von Hansjörg Siegenthaler. ${ }^{26}$ In Bezug auf das 19. Jahrhundert fokussierte die Forschung seit längerem auf die Bedeutung wirtschaftlicher Faktoren für die Bundesstaatsgründung und der Bundesverfassung von 1848 für den wirtschaftlichen Erfolg der Schweiz.

Dabei ist bis heute kein Konsens unter den Historikerinnen und Historikern zu erkennen. Während Cédric Humair den Einfluss von wirtschaftlichen Faktoren auf die Bundesstaatsgründung als hoch veranschlagt, sind Hansjörg Siegenthaler sowie Patrick Halbeisen und Margrit Müller skeptisch.. ${ }^{27}$ Die Argumente, die den beiden Interpretationen zugrunde liegen, sind gut nachvollziehbar. Humair weist auf die zunehmende wirtschaftliche Integration infolge der Industrialisierung und der Kommunikations- und Transportrevolution hin, die in den 1840er Jahre an Tempo gewann. Dagegen hebt Siegenthaler hervor, dass sich der Sonderbund an den "Quellen der Wahrheit» entzündet habe. Wirtschaftliche Themen hätten nicht im Zentrum der Auseinandersetzung zwischen Radikalen und Katholisch-Konservativen gestanden, sondern die Frage, inwieweit die katholische Kirche das Bildungswesen bestimmen und die Loyalität zu den eidgenössischen Institutionen in Frage stellen darf. Halbeisen und Müller

25 Die bisher einzige Studie beschäftigt sich mit der ersten Hälfte des 20. Jahrhunderts: Roman Wild, Auf Schritt und Tritt: Der schweizerische Schuhmarkt 1918-1948, Zürich 2019.

26 Hansjörg Siegenthaler, Regelvertrauen, Prosperität und Krisen. Die Ungleichmässigkeit wirtschaftlicher und sozialer Entwicklung als Ergebnis individuellen Handelns und sozialen Lernens, Tübingen 1993. Vgl. die Diskussion der institutionellen Faktoren bei James Breiding und Gerhard Schwarz, Wirtschaftswunder Schweiz. Ursprung und Zukunft eines Erfolgsmodells, Zürich 2011.

27 Hansjörg Siegenthaler, Supranationalität, Nationalismus und regionale Autonomie: Erfahrungen des schweizerischen Bundesstaates - Perspektiven für die europäische Gemeinschaft, in: Traverse (1994), S. 117-140; Patrick Halbeisen und Margrit Müller, Ökonomische Motive und Erwartungen - ihr Einfluss auf die Bundestaatsgründung, in: Andreas Ernst, Albert Tanner, Matthias Weishaupt (Hg.), Revolution und Innovation: Die konfliktreiche Entstehung des schweizerischen Bundesstaates von 1848, Zürich 1998, S. 117-136. 
zeigen, dass man wirtschaftliche Interessen auch auf anderem Wege hätte institutionell berücksichtigen können, zum Beispiel durch kantonale Zollrevisionen, freiwillige interkantonale Vereinbarungen (Konkordate), Beschlüsse der Tagsatzung und eine Teilrevision des Bundesvertrags von 1815.

Ein ähnlicher Dissens existiert auch im Hinblick auf die Frage, inwiefern die Bundesverfassung von 1848 das Wirtschaftswachstum begünstigt hat. Joseph Jung spricht von einem günstigen «liberalen Zeitfenster» bis zur ersten Verfassungsrevision von 1874, als das Referendumsrecht eingeführt wurde. Die Schaffung eines Binnenmarkts und die Klärung der institutionellen Frage hätten zu einem enormen wirtschaftlichen Schub geführt, was sich unter anderem in der Anzahl der Firmengründungen niedergeschlagen habe. Dabei habe vor allem der Zürcher Politiker und Unternehmer Alfred Escher eine entscheidende Rolle gespielt. ${ }^{28}$ Béatrice Veyrassat hingegen argumentiert, dass die Referendumsdemokratie die wirtschaftliche Entwicklung genauso positiv begünstigt habe, da sie für eine enorme innenpolitische Stabilität gesorgt habe. Es sei durch den Ausbau der Volksrechte gut gelungen, eine fein austarierte Balance zwischen Wirtschaftsliberalismus, Demokratie und staatlicher Regulierung zu finden. ${ }^{29}$

Auch hier gilt, dass die Forschung bisher noch zu wenig einzelne Regionen und Branchen untersucht hat, um in dieser Frage weiterzukommen. Bis weit ins 20. Jahrhundert war ja der Bundesstaat relativ zurückhaltend, was die Regulierung der Wirtschaft anbelangte, und die Stabilität der eidgenössischen Politik hing nicht nur mit der Bundesverfassung oder dem Referendumsrecht auf Bundesebene zusammen, sondern ebenso mit dem politischen Ausgleich in und zwischen den Kantonen und Gemeinden. Für einen Grossbetrieb war es in vielerlei Hinsicht wichtiger, die kommunale Politik zu dominieren, als die Bundespolitik zu beeinflussen. Die Aussenhandelspolitik wurde im Wesentlichen von den Wirtschaftsverbänden formuliert und umgesetzt, während der Bund nur unterstützend wirkte. Will man also die Bedeutung der Institutionen für das Wirtschaftswachstum beschreiben, muss man das gesamte Geflecht zwischen Firmen, Branchenverbänden, Parteien und Behörden von unten nach oben untersuchen. In vielen unternehmensgeschichtlichen Arbeiten wird die Rolle der Politik erwähnt, aber es existieren kaum systematische Studien in der Art, wie es zum Technik, Band 100, Zürich 2013; Joseph Jung (Hg.), Alfred Escher. Briefe. Ein Editions- und Forschungsprojekt der Alfred Escher-Stiftung, 6 Bände, Zürich 2008-2015. Digitale Version (seit 2015 verfügbar): www.briefedition.alfred-escher.ch (12.01.2021); Joseph Jung, Das Laboratorium des Fortschritts. Die Schweiz im 19. Jahrhundert, Zürich 2019.

29 Béatrice Veyrassat, Wirtschaft und Gesellschaft an der Wende zum 20. Jahrhundert, Basel 2012, S. 33-81. Siehe auch Thomas David und André Mach, Institutions and Economic Growth. The Successful Experience of Switzerland (1870-1950), in: Unu-Wider Research Paper 101 (2006). 
Beispiel André Kienzle für die Zwischenkriegszeit am Beispiel der Von Roll in Gerlafingen vorgemacht hat. ${ }^{30}$

\section{Kultur und Bildung}

Die Frage nach der Bedeutung von Institutionen ist von derjenigen nach der Rolle der Kultur nicht zu trennen. Wenn ein Konsens über die wesentlichen Werte fehlt, sind Institutionen nicht funktionstüchtig, und umgekehrt hängt der Konsens von der Ausgestaltung der Institutionen ab. Wenn zentrale Werte wie zum Beispiel die rechtliche Gleichberechtigung nicht institutionell abgesichert werden, ist die Politik gelähmt. In der Schweiz ist es fast immer gelungen, Spannungen zwischen Werten und Institutionen auf friedliche Weise abzubauen. Selbst der Sonderbundskrieg von 1847 und der Landesstreik von 1918 waren im Vergleich zu anderen Bürgerkriegen des 19. Jahrhunderts und den Revolutionen nach Ende des Ersten Weltkriegs harmlos.

Kultur ist auch wirtschaftlich von grosser Bedeutung. Wie genau die Wirkungszusammenhänge zu verstehen sind, ist spätestens seit der Publikation von Max Webers Studie «Die protestantische Ethik und der Geist des Kapitalismus» (1904/05) höchst umstritten. Angestossen vom einflussreichen Aufsatz von Sascha Becker und Ludger Wössmann scheint sich jedoch eine gewisse Einigkeit in Bezug auf die Bildung als wichtigster Transmissionskanal eingestellt zu haben. ${ }^{31}$ Der Protestantismus sah es ja als wichtig an, dass die Gläubigen die Bibel selber lesen konnten, was Bemühungen um eine Alphabetisierung auslöste. Becker und Wössmann können für Preussen eine klare Kausalität nachweisen.

Die Schweiz ist für diese Forschungsdebatte besonders interessant, weil für das 19. Jahrhundert eine auffällige Konzentration der Industrialisierung auf die reformierten Gebiete beobachtet werden kann. Geradezu verblüffend ist, wie verschieden die Verhältnisse auf engstem Raum waren. Während zum Beispiel der Kanton Appenzell Ausserrhoden äusserst stark industrialisiert war, dominierte im Kanton Appenzell Innerrhoden noch am Ende des 19. Jahrhundert die Landwirtschaft. Die Konfession scheint also durchaus relevant gewesen zu sein, aber noch ist unklar, ob deren Einfluss vor allem über die Bildung zum Tragen kam oder über einen anderen Mechanismus. Ein kliometrischer Aufsatz aus dem Jahr

30 André Kienzle, «Es gibt nur ein Gerlafingen». Herrschaft, Kultur und Soziale Integration in einer Standortgemeinde des Stahlkonzerns Von Roll 1918-1939, Zürich 1997. Vgl. die neue Studie von Markus Somm, Elektropolis an der Limmat. Baden und die BBC, 1870 bis 1925 - Die Beschreibung einer Transformation, Bern 2019.

31 Sascha O. Becker und Ludger Wössmann, Was Weber Wrong? A Human Capital Theory of Protestant Economic History, in: The Quarterly Journal of Economics 124 (2009), S. 531-596. Einen interessanten Überblick über dieses dynamische Forschungsfeld bieten Sascha O. Becker, Jared Rubin und Ludger Wössmann, Religion in Economic History: A Survey, in: CEPR Discussion Paper 14894 (2020). 
2013, der die stark divergierenden Ergebnisse der Rekrutenprüfungen zu erklären versucht, kommt zum Schluss, dass das Bildungsniveau nur zum Teil mit der Konfessionszugehörigkeit erklärt werden kann. Ebenso wichtig für den Bildungsstand eines Bezirks am Ende des 19. Jahrhunderts sei gewesen, ob es sich um eine ländliche oder städtische Gegend handelte. ${ }^{32}$

Der Aufsatz macht aber auch deutlich, dass die Bildungsforschung in der Wirtschaftsgeschichte bis heute noch wenig entwickelt ist. ${ }^{33}$ Dabei kann das 19. Jahrhundert ohne Übertreibung als das Jahrhundert der Bildung bezeichnet werden. Die grosse Leistung der Liberalen war die nahezu vollständige Alphabetisierung bis zum Ende des Jahrhunderts. Nicht nur die heutigen Volksschulen, Gymnasien und Hochschulen, sondern auch die Berufsschulen wurden damals gegründet. Seit 1884 vom Bund subventioniert und reguliert, leisteten sie einen entscheidenden Beitrag zur Ausbildung der Facharbeiterschaft und des kaufmännischen Personals und erhöhten die Chancen der schweizerischen Volkswirtschaft, den rasanten Strukturwandel zu bewältigen. Vorteilhaft war mit Sicherheit die dezentrale Struktur des Bildungssystems, denn sie erlaubte eine enge Verzahnung mit den lokalen und regionalen wirtschaftlichen Interessen.

Auf der anderen Seite darf man die Bedeutung der formalen Bildung nicht überschätzen. Der Anteil der Ungelernten und Angelernten blieb durch das ganze 19. Jahrhundert hindurch hoch, und das Lernen am Arbeitsplatz dürfte selbst für diejenigen, die über eine Tertiärausbildung verfügten, weiterhin sehr wichtig gewesen sein. Arbeitsabläufe und technisches Wissen wurden zu einem grossen Teil durch Routine eingeübt. Über die Rolle der Ungelernten und des praktischen Lernens, das oft mündlich erfolgte, wissen wir so gut wie nichts. Hier liegt ein grosses offenes Forschungsfeld vor uns.

\section{Banken und Industrialisierung}

In ihrem fortgeschrittenen Stadium benötigte die Industrialisierung im 19. Jahrhundert ein modernes Bankensystem, das die Spargelder bündelte und in die kapitalintensiven Branchen lenkte. Den Startschuss hierzu gab der Eisenbahnbau, der in den 1850er Jahren einsetzte. Die klassische Eisenbahnbank war die Schweizerische Kreditanstalt, die 1856 in Zürich gegründet wurde und als Finanzierungsvehikel der Nordostbahn diente. In Basel kümmerten sich die traditionellen Privatbanken um die Finanzierung der Centralbahn, der zweitwichtigsten

32 Timo Boppard, Josef Falkinger, Volker Grossmann, Ulrich Woitek und Gabriela Wüthrich, Under Which Conditions Does Religion Affect Educational Outcomes?, in: Explorations in Economic History 50 (2013), S. 242-266.

33 Dies gilt nicht für die allgemeine Bildungsforschung. Ein grosser Meilenstein ist beispielsweise die 2015 abgeschlossene Herausgabe der Stapfer'schen Schul-Enquête (Helvetische Schulumfrage von $1799)$ und die daran anschliessenden Forschungsarbeiten: www.stapferenquete.ch (12.01.2021). 
Eisenbahngesellschaft der Schweiz. In der Westschweiz waren es hauptsächlich französische Banken wie der Crédit Mobilier, die das Investitionskapital zur Verfügung stellten. Diese Geschichte ist weitgehend bekannt. ${ }^{34}$

Weniger bekannt sind die Beziehungen zwischen den Banken und den einzelnen Industriebetrieben. In Unternehmenschroniken wird meist nur am Rande erwähnt, wie die zentralen Verbindungen zu den «Hausbanken» entstanden und sich veränderten, so dass eine systematische Analyse der Kreditgeber und ihrer Vertreter in den Verwaltungsräten weitgehend fehlt. Die Rekonstruktion der Finanzbeziehungen ist eine äusserst aufwändige Arbeit, weil man alle Verwaltungsratsprotokolle und Kreditbücher, sofern sie vorhanden sind, durchforsten muss. Der Aufwand würde sich lohnen, um besser zu verstehen, wie die verschiedenen Netzwerke der Schweizer Wirtschaft im 19. Jahrhundert entstanden und funktionierten.

Die Untersuchung würde ferner auch zeigen, warum sich in der Ostschweiz und in der Westschweiz keine Grossbanken dauerhaft etablieren konnten. Denn nur wenn ein grosser Kapitalbedarf ausgewiesen war, was die Banken zu Kreditund Emissionsaufträgen verhalf, konnte sich ein grösseres Institut herausbilden. Dafür war St. Gallen der Ort, von dem aus die Privatversicherungen der Schweiz aufgebaut wurden, während die beiden führenden Finanzplätze Basel und Zürich erst mit einer gewissen Verzögerung diesen Teil des Finanzsektors entwickelten. Die Ursachen dieser unterschiedlichen Verlaufsprozesse sind nie gesamtschweizerisch analysiert worden.

Schliesslich müsste eine Untersuchung der Kreditgeschichte des 19. Jahrhunderts auch die Frage zu beantworten versuchen, wie stark bestimmte Regionen, Firmen und Haushalte unter einer Kreditknappheit litten und inwiefern Kantonalbanken und Raiffeisenbanken in der Lage waren, diese Knappheit zu überwinden. Abgesehen von einzelnen Beispielen und zeitgenössischen Äusserungen von Politikern haben wir bisher kaum empirische Evidenz, die das Ausmass der Kreditknappheit nur annähernd quantifizieren könnte. Je nach Region dürfte die Antwort unterschiedlich ausfallen. ${ }^{35}$

34 Youssef Cassis, L'histoire des banques suisses aux XIX et XX ${ }^{e}$ siècles, in: Schweizerische Zeitschrift für Geschichte 41 (1991), S. 512-520; Youssef Cassis und Jakob Tanner (Hg.), Banken und Kredit in der Schweiz (1850-1930), Zürich 1993. Neuere Forschungen: Pietro Nosetti, Le secteur bancaire tessinois. Origines, crises et transformations (1861-1939), Neuchâtel 2018.

35 Mischa Suter, Rechtstrieb. Schulden und Vollstreckung im liberalen Kapitalismus 1800-1900, Konstanz 2016. 


\section{Gewerbe und Kleinbetriebe}

Im 19. Jahrhundert waren die kleinen und mittelgrossen Unternehmen (KMU) die zahlenmässig grösste Gruppe der Arbeitgeber. Dies bedeutet nicht, dass die Grossunternehmen weniger bedeutend als die KMU waren. Auch wäre es falsch, die eine Unternehmensgruppe gegen die andere auszuspielen, denn sie waren auf verschiedene Weise miteinander verflochten. Aber für die Arbeitsrealität der meisten Beschäftigten war der Kleinbetrieb die prägende Erfahrung. Zumal in den Städten war die Dichte an Gewerbebetrieben äusserst hoch.

$\mathrm{Zu}$ dieser Thematik ist in jüngerer Zeit kaum geforscht worden, obwohl eine Reihe von wichtigen Forschungsfragen vorhanden ist. ${ }^{36} \mathrm{Zu}$ untersuchen wäre insbesondere, wie die verschiedenen Gewerbezweige von der Industrialisierung betroffen waren. Falsch ist sicherlich die Vorstellung, dass sie alle von der Fabrikindustrie zunehmend an den Rand gedrängt wurden. Für einige mag dies zutreffen, aber viele andere dürften von der Industrialisierung enorm profitiert haben, insbesondere das Gastgewerbe, die Hotellerie, der Tourismus, der Detailhandel, die Bauwirtschaft und verwandte Branchen, die zum Aufbau der Infrastruktur beitrugen, aber auch zahllose spezialisierte Zulieferbetriebe. Die Entwicklung verlief alles andere als einförmig.

Zweitens wäre es lohnend, die sich ändernden rechtlichen Rahmenbedingungen zu untersuchen. Bis in die 1830er Jahre regierten mancherorts Zünfte das handwerkliche Gewerbe. Darauf fielen diese jahrhundertealten Schranken weg, was viele geschäftliche Möglichkeiten eröffnete, vor allem für Aussenseiter, die bisher ausgeschlossen worden waren. Aber es herrschte nun keineswegs ein freier, ungezügelter Wettbewerb, denn die grösseren Betriebe und die sich formierenden Interessenverbände begannen bald ihre Dominanz mit anderen Mitteln abzusichern. Aufträge konnten politisch erwirkt werden, oder eine Branche setzte sich für Vorschriften ein, die nur ein Teil der Konkurrenten erfüllen konnte. $\mathrm{Zu}$ dieser Art von Wettbewerbsbarrieren existieren kaum Studien.

Eine dritte Frage betrifft die Rolle der Familie in den Gewerbebetrieben. Oft waren Wohnort und Arbeitsort kaum getrennt, die Frau des Geschäftsinhabers spielte oft eine wichtige geschäftliche Rolle, und die Kinder leisteten einen Teil der Arbeit. Dies erlaubte es den Gewerbebetrieben, die Lohnkosten flexibel zu gestalten und damit auch schnell auf grössere Konjunkturverwerfungen zu reagieren. Es ist methodisch schwierig, die Bedeutung der Familie wirtschaftlich genauer zu erfassen, weil private Quellen zu diesem Thema nur spärlich öffentlich zugänglich sind. Aber zumindest qualitativ dürfte es möglich sein, einen besseren Einblick in die Alltagswelt des Gewerbes zu gewinnen.

36 Zum Beispiel Peter Haenger, Das Fleisch und die Metzger. Fleischkonsum und Metzgerhandwerk in Basel seit der Mitte des 19. Jahrhunderts, Zürich 2001. 


\section{Die Bedeutung des Sozialkapitals}

Wirtschaftlicher Erfolg ist nur möglich, wenn die verschiedenen Gruppen und Individuen, die dazu beitragen, ein gewisses gegenseitiges Vertrauen besitzen, das heisst wenn Sozialkapital vorhanden ist. ${ }^{37}$ Nach allem, was wir wissen, scheint die Schweizer Gesellschaft im 19. Jahrhundert ein sehr hohes Mass davon besessen zu haben. Die Menschen haben sich politisch beteiligt, Vereine gegründet, Milizarbeit geleistet, Zeitungen gelesen und gemeinsam Feste gefeiert. Es bestand ein dichtes Netz an Beziehungen innerhalb der Milieus, aber auch zwischen den Milieus, besonders auf kommunaler Ebene.

Wie die schweizerische Bürgertumsforschung herausgearbeitet hat, war die schweizerische Wirtschaftselite recht offen für Aufsteiger aus dem Mittelstand. ${ }^{38}$ Die politische Elite war sogar ausgesprochen durchlässig - und ist es bis heute. Zwar gab es auch in der Schweiz im späten 19. Jahrhundert Abgrenzungsstrategien - kontrollierte Heiratskreise, exklusive Gesellschaften und das Offizierkorps etc. -, aber die soziale Durchmischung der Elite fand dennoch statt, besonders als neue Branchen wie die Chemie-, Elektro- und Maschinenindustrie enorme Karrieren ermöglichten.

Die Entwicklung des schweizerischen Sozialkapitals im 19. Jahrhundert ist noch nie richtig erforscht worden. Vor allem wissen wir sehr wenig über den gewerblichen und bäuerlichen Mittelstand, der in den Kleinstädten und ländlichen Gebieten eine Schlüsselrolle einnahm. $\mathrm{Zu}$ untersuchen wäre zum Beispiel die Rolle der Wirtshausbesitzer, die nicht nur als Gastgeber, sondern auch als Politiker grossen Einfluss hatten. Aus dem Milieu des gewerblichen und bäuerlichen Mittelstandes stammten auch viele Aufsteiger, die über den Besuch des Eidgenössischen Polytechnikums (ab 1905 ETH) und eines Technikums (Biel, Burgdorf, Winterthur) in die Ränge der höheren Angestellten in Privatunternehmen aufstiegen. Die Gruppe ist gross und heterogen, aber bisher kaum angemessen berücksichtigt worden. Bei diesem Thema empfiehlt es sich, regionale und lokale Studien mit quantitativen Erhebungen zu kombinieren. Dazu dürften in den Staats- und Gemeindearchiven, Lokalzeitungen und Hochschularchiven genügend Quellen existieren, die es ermöglichen, den Mikrokosmos des gewerblichen und bäuerlichen Mittelstands besser zu erschliessen und zu beschreiben. Es würde das bisherige Bild, das von der Erforschung der Oberschicht und der

37 Dazu grundlegend der Hans Ulrich Jost und Albert Tanner, Geselligkeit, Sozietäten und Vereine, in: Schweizerisches Jahrbuch für Wirtschafts- und Sozialgeschichte 9 (1991).

38 Albert Tanner, Arbeitsame Patrioten - wohlanständige Damen. Bürgertum und Bürgerlichkeit in der Schweiz 1830-1914, Zürich 1995. 
Führungsschicht der Arbeiterbewegung geprägt ist, gut ergänzen und damit einen wesentlichen Beitrag zur Erforschung des Sozialkapitals im 19. Jahrhundert leisten.

Tobias Straumann, Universität Zürich, Zürichbergstrasse 14, 8032 Zürich, tobias.straumann@econ.uzh.ch 